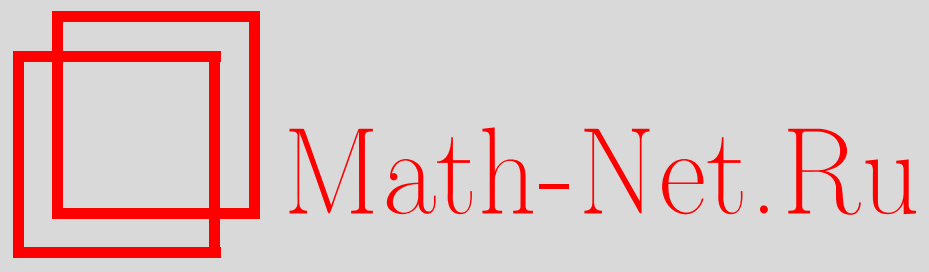

H. Б. Безверхняя, Гиперболичность некоторых 2порожденных групп с одним определяющим соотношением, Дискрет. матем., 2002, том 14, выпуск 3, 54-69

DOI: https://doi.org/10.4213/dm254

Использование Общероссийского математического портала Math-Net.Ru подразумевает, что вы прочитали и согласны с пользовательским соглашением http://www . mathnet.ru/rus/agreement

Параметры загрузки:

IP : 3.91 .87 .62

26 апреля 2023 г., 14:02:31

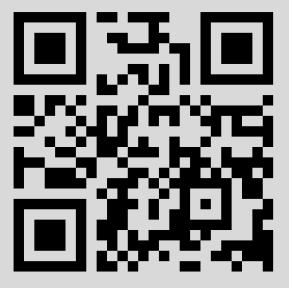




\title{
Гиперболичность некоторых 2-порожденных групп с одним определяющим соотношением
}

\author{
(ㄷ) 2002 г. Н. Б. Безверхняя
}

\begin{abstract}
В статье дается описание словарно гиперболических групп, которые имеют представление$$
\left\langle a, b, t ; t^{-1} a t=b, t^{-1} b t=w(a, b)\right\rangle .
$$

Работа выполнена при поддержке Российского фонда фундаментальных исследований, грант 00-01-00767.
\end{abstract}

Пусть $G\langle A ; R\rangle$ - конечнопредставленная группа, слово $W$ в алфавите $A^{ \pm 1}$ равно единице в групте $G$ тогда и только тогда, когда

$$
W=\prod_{i=1}^{n} S_{i} R_{i}^{ \pm 1} S_{i}^{-1}
$$

в свободной группе $F=F(A)$, где $S_{i} \in F$ и $R_{i} \in R$.

Группа $G$ называется (словарно) гиперболической, если существует функция $n=n(W)$, ограничивающая минимальное число сомножителей в (1), линейно зависящая от длины $W$.

Вопрос об определении класса гиперболических групп среди всех групп с одним определяющим соотношением является весьма важным, так как гиперболические группы обладают рядом свойств таких, как разрешимость проблемы сопряженности слов [1], обобщенной сопряженности слов [2], $S Q$-универсальности [3], разрешимости квадратичных уравнений $[1,2]$. В [4] выделен некоторый подкласс гиперболических групп с одним определяющим соотношением. И. Капович в [5] доказал гиперболичность групп

$$
G=\left\langle a, t ; t^{2} a t^{-1} a t a^{2} t^{-2} a^{-1}=1\right\rangle .
$$

Автором из множества групп с одним определяющим соотношением выделен подкласс гиперболических груп, не совпадающий с подклассом гиперболических групп из [4] и содержащий группу Каповича. Автор рассматривает 2-порожденные группы с одним определяющим соотношением, заданные как HNN-расширение некоторой свободной группы ранга 2.

Определим следующие понятия. Пусть

$$
G^{*}=\left\langle G, t ; t^{-1} \alpha_{-1}(c) t=\alpha_{1}(c), c \in C\right\rangle
$$

- HNN-расширение группы $G$, соответствующее мономорфизмам $\alpha_{-1}, \alpha_{1}: C \rightarrow G$ для некоторой группы $C$. 
Определение 1 ([5]). Аннулятором длины $2 M+1$ в (2) будем называть пару $\Sigma=(\bar{p}, \bar{c})$, где

$$
\bar{p}=\left(t^{\varepsilon_{-M}}, h_{-M}, t^{\varepsilon_{-M+1}}, h_{-M+1}, \ldots, t^{\varepsilon_{0}}, h_{0}, t^{\varepsilon_{1}}, \ldots, t^{\varepsilon_{M-1}}, h_{M-1}, t^{\varepsilon_{M}}\right),
$$

все $h_{i}$ для $i \in\{-M, \ldots, M-1\}$ принадлежат группе $G, \varepsilon_{i}= \pm 1$,

$$
\bar{c}=\left(c_{-M}, c_{-M+1}, \ldots, c_{0}, c_{1}, \ldots, c_{M}\right),
$$

все $c_{i} \in C$ для $i \in\{-M, \ldots, M\}$, причем пара $\Sigma(\bar{p}, \bar{c})$ должна удовлетворять следующему условию: для любого $i \in\{-M, \ldots, M-1\}$

$$
h_{i}^{-1} \alpha_{\varepsilon_{i}}\left(c_{i}\right) h_{i}=\alpha_{-\varepsilon_{i+1}}\left(c_{i+1}\right),
$$

то есть

$$
(p)^{-1} \alpha_{-\varepsilon_{-M}}\left(c_{-M}\right) p=\alpha_{\varepsilon_{M}}\left(c_{M}\right),
$$

где

$$
p=\left(t^{\varepsilon_{-M}} h_{-M} t^{\varepsilon_{-M+1}} h_{-M+1} \ldots t^{\varepsilon_{0}} h_{0} t^{\varepsilon_{1}} \ldots t^{\varepsilon_{M-1}} h_{M-1} t^{\varepsilon_{M}}\right) .
$$

Аннулятор $\Sigma$ называется существенным, если $\bar{p}$ не содержит подпоследовательностей вида $t^{-1}, \alpha_{-1}(c), t$ или $t, \alpha_{1}(c), t^{-1}$.

Определение 2 ([5]). Пусть $G^{*}$ имеет копредставление (2), $G$ и $C$-конечнопорожденные группы. Обозначим $l_{C}, l_{G}$ и $l_{G^{*}}-$ функции длины соответственно в группах $C, G$ и $G^{*}$.

Пусть $\Sigma=(\bar{p}, \bar{c})$ - аннулятор длины $2 M+1$, тогда $l_{C}\left(c_{0}\right)$ будем называть обхватом аннулятора, а $\max -M \leqslant i \leqslant M-1\left(l_{G}\left(h_{i}\right)\right)$ его шириной.

Если существует действительное число $\lambda>1$ такое, что

$$
\lambda l_{C}\left(c_{0}\right)<\max \left(l_{C}\left(c_{-M}\right), l_{C}\left(c_{M}\right)\right),
$$

то аннулятор называется $\lambda$-гиперболическим.

Определение 3 ([5]). Пусть $G$ - конечнопорожденная группа, $X$ - конечное множество ее образующих и $l_{X}$ - словарная функция длины в $G$. Пусть $H$ - конечнопорожденная подгруппа группы $G$ и $Y$ - множество образующих группы $H$ и $l_{Y}$ - словарная функция длины в $H$. Будем говорить, что $H$ квазивыпукла в $G$, если существует $c>0$ такое, что для любого $h \in H$

$$
\frac{1}{c} l_{X}(h) \leqslant l_{Y}(h) \leqslant c l_{X}(h) .
$$

В дальнейшем при доказательстве используется комбинаторная теорема Бествины $[6,7]$.

Теорема 1 ([6,7]). Пусть $G$ - гиперболическая конечнопорожденная группа, и пусть $\alpha_{-1}, \alpha_{1}: C \rightarrow G-$ мономорфизмы такие, что подгруппы $\alpha_{-1}(C), \alpha_{1}(C)$ квазивыпуклы в $G, G^{*}-H N N$-расширение $G$,

$$
C^{*}=\left\langle G, t ; t^{-1} \alpha_{-1}(c) t=\alpha_{1}(c), c \in C\right\rangle .
$$

Если существует действительное число $\lambda>1$ и челое $M>0$ такие, что для произвольного $\rho>0$ существует $H(\rho)$ такое, что всякий существенный аннулятор длины $2 M+1$ c шириной не более $\rho$ и обхватом не менее $H(\rho)$ является $\lambda$-гиперболическим, тогда $G^{*}$ - гиперболическая группа. 
Теорема 2 ([8]). Пусть

$$
G^{*}=\left\langle a, b, t ; t^{-1} a t=b, t^{-1} b t=w\right\rangle
$$

- HNN-расширение свободной группы $G=\langle a, b\rangle$. Группа $G^{*}$ является гиперболической, если

$$
\begin{aligned}
& w \neq a x b^{k_{1}} x^{-1} a^{-1}, \\
& w \neq \equiv x a^{k_{2}} x^{-1} a^{-1}, \\
& w \neq a^{\varepsilon} x\left(a^{\mu} b^{\delta}\right)^{p} x^{-1} a^{-\varepsilon} b^{-\delta \mu},
\end{aligned}
$$

$\delta= \pm 1, \varepsilon= \pm 1, p \in \mathbf{N} u w \neq a^{m} b^{n}$, где либо $|m|=1$, либо $|m|=|n| \pm 1$.

Теорема 3. Пусть

$$
G^{*}=\left\langle a, b, t ; t^{-1} a t=b, t^{-1} b t=w\right\rangle
$$

- HNN-расширение свободной группь $G=\langle a, b\rangle$, тогда $G^{*}$ не является гиперболической, если либо $w=a x b^{k_{1}} x^{-1} a^{-1}$, либо $w=a x a^{k_{2}} x^{-1} a^{-1}$, либо

$$
w=a^{\varepsilon} x\left(a^{\mu} b^{\delta}\right)^{p} x^{-1} a^{-\varepsilon} b^{-\delta \mu}, \quad \delta= \pm 1, \quad \varepsilon= \pm 1, \quad p \in \mathbf{N},
$$

либо $w=a^{m} b^{n}$, где или $|m|=1$, или $|m|=|n| \pm 1$.

Доказательство теоремы 2. Предварительно рассмотрим ряд вспомогательных результатов.

Определение 4 ([9]). Подгруппа $H$ группы $G$ антинормальна в $G$, если для любого $g \in G \backslash H$

$$
g^{-1} H g \cap H=E,
$$

где $E$ - единичная подгруппа.

Лемма 1 ([5]). Пусть

$$
G^{*}=\left\langle G, t ; t^{-1} \alpha_{-1}(c) t=\alpha_{1}(c), c \in C\right\rangle
$$

и $\alpha_{-1}(C), \alpha_{1}(C)$ антинормальны в $G$, тогда не существует аннулятора $\Sigma=(\bar{p}, \bar{c})$ такого, что $\bar{p}$ содержит подпоследовательность вида $t^{\varepsilon}, h, t^{-\varepsilon}$, где $\varepsilon= \pm 1, h \in G$, то есть всякий существенный аннулятор однонаправлен.

Пусть $f$-слово свободной группы $F$, обозначим $f^{*}$ его произвольную циклическую перестановку.

Лемма 2 ([10]). Пусть $F=\left\langle a_{1}, a_{2}, \ldots, a_{n}\right\rangle$ - свободная группа, $a_{1}, a_{2}, \ldots, a_{n}-$ ее свободные образующие, $H=\left\langle a_{1}, \ldots, a_{k}, f\left(a_{1}, \ldots, a_{n}\right)\right\rangle$ - собственная подгруппа группы $F$ такая, что $k<n$ и $f^{*} \notin\left\langle a_{1}, \ldots, a_{k}\right\rangle$. Тогда, если с минимален в $H c H, H c H \neq H Н u$ cH $c^{-1} \cap H \neq E$, то $c H c^{-1} \cap H-$ чиклическая подгруппа.

Следствие 1 ([10]). Пусть $H=\langle b, w\rangle$ - собственная подгруппа ранга 2 группы $\langle a, b\rangle$. Если для $h \in\langle a, b\rangle$, минимального в $H h H, H h H \neq H H, h H h^{-1} \cap H-$ чиклическая подгруппа, то либо $w=v^{p}$ для некоторого $p \in \mathbf{Z} \backslash\{0\}, h H h^{-1} \cap H=\left\langle v^{p}\right\rangle u h v^{p} h^{-1}=v^{p}$, либо существует такое $k \in \mathbf{Z} \backslash\{0\}$, что $w b^{k}=v^{p}, h H h^{-1} \cap H=\left\langle v^{p}\right\rangle u h\left(w b^{k}\right)^{s} h^{-1}=$ $\left(b^{k} w\right)^{s}$, либо $h\left(w b^{k}\right)^{s} h^{-1}=\left(w b^{k}\right)^{s}, s \in \mathbf{Z} \backslash\{0\}$. 
Лемма 3. Пусть $F_{n}=\left\langle a_{1}, a_{2}, \ldots, a_{n}\right\rangle-$ свободная группа,

$$
H=\left\langle a_{1}, \ldots, a_{k}, f\left(a_{1}, \ldots, a_{n}\right)\right\rangle, \quad k<n,
$$

- noдгpynna гpynnы $F_{n}, f\left(a_{1}, a_{2}, \ldots, a_{n}\right) \notin\left\langle a_{1}, \ldots, a_{k}\right\rangle$ u $f=s f_{0} s^{-1}$, где $f_{0} \in\left\langle a_{1}, \ldots, a_{k}\right\rangle, s=a_{\nu}^{\varepsilon_{1}} s_{0} a_{\mu}^{\varepsilon_{2}}, v, \mu \notin\{1, \ldots, k\}, \varepsilon_{1}, \varepsilon_{2} \in\{ \pm 1\}$.

Тогда

$$
s H s^{-1} \cap H=\langle f\rangle, \quad s^{-1} H s \cap H=\left\langle f_{0}\right\rangle .
$$

Лемма 4. Пусть $F_{n}=\left\langle a_{1}, a_{2}, \ldots, a_{n}\right\rangle-$ свободная группа,

$$
H=\left\langle a_{1}, \ldots, a_{k}, f\left(a_{1}, \ldots, a_{n}\right)\right\rangle, \quad k<n,
$$

- noдгруnпа группы $F_{n}, f\left(a_{1}, a_{2}, \ldots, a_{n}\right) \notin\left\langle a_{1}, \ldots, a_{k}\right\rangle$ u $f=s^{-1} f_{0}^{p} s$, где $s=a_{v}^{\varepsilon_{1}} s_{0} a_{\mu}^{\varepsilon_{2}}, v, \mu \notin\{1, \ldots, k\}, \varepsilon_{i}= \pm 1, i \in\{1,2\},|p|>1 u f_{0} \in\left\langle a_{1}, \ldots, a_{k}\right\rangle$.

Тогда

$$
\left(s^{-1} f_{0}^{-l} s\right) H\left(s^{-1} f_{0}^{l} s\right) \cap H=\langle f\rangle,
$$

где $l \in\{1, \ldots, p-1\}$.

Лемма 5. Пусть $F_{n}=\left\langle a_{1}, a_{2}, \ldots, a_{n}\right\rangle-$ свободная группа,

$$
H=\left\langle a_{1}, \ldots, a_{k}, f\left(a_{1}, \ldots, a_{n}\right)\right\rangle, \quad k<n,
$$

- noдгpynna грynnы $F_{n}, f\left(a_{1}, a_{2}, \ldots, a_{n}\right) \notin\left\langle a_{1}, \ldots, a_{k}\right\rangle$ u $f=s f_{0}^{p} s^{-1}$, где $f_{0} \in\left\langle a_{1}, \ldots, a_{k}\right\rangle, s=a_{v}^{\varepsilon_{1}} s_{0} a_{\mu}^{\varepsilon_{2}}, v, \mu \notin\{1, \ldots, k\}, \varepsilon_{i}= \pm 1, i \in\{1,2\},|p|>1$ u nycmb $z \in F_{n}$ такой, что $z$-минимален в $H z H, H z H \neq H H u z \neq s^{ \pm 1}, z \neq s^{-1} f_{0}^{l} s$ nрu $|p|>1$, mогда $z^{-1} H z \cap H=E$.

Доказательства лемм 3-5 проводятся непосредственным вычислением.

Лемма 6. Пусть $F_{n}=\left\langle a_{1}, a_{2}, \ldots, a_{n}\right\rangle-$ свободная группа,

$$
H=\left\langle a_{1}, \ldots, a_{k}, f\left(a_{1}, \ldots, a_{n}\right)\right\rangle, \quad k<n,
$$

- nодгруnпа групnы $F_{n}, f\left(a_{1}, a_{2}, \ldots, a_{n}\right) \notin\left\langle a_{1}, \ldots, a_{k}\right\rangle, f=f_{0} v$, где $f_{0}=a_{v}^{\varepsilon_{1}} \bar{f}_{0} a_{\mu}^{\varepsilon_{2}}$, $\nu, \mu \notin\{1, \ldots, k\}, \varepsilon_{i}= \pm 1, i \in\{1,2\}, u v \in\left\langle a_{1}, \ldots, a_{k}\right\rangle$. Пусть для некоторого $z \in F_{n}$, минимального в НzНи удовлетворяющего условию $\mathrm{HzH} \neq \mathrm{HH}$, и для некоторого $w_{0} \in\left\langle a_{1}, \ldots, a_{k}\right\rangle$, справедливо включение $z^{-1} w_{0} z \in H$, тогда $z^{-1} w_{0} z=f_{0}^{p} u$ $f_{0} \equiv s^{-1} \tilde{f}_{0} s$, где $\tilde{f}_{0} \in\left\langle a_{1}, \ldots, a_{k}\right\rangle, s=a_{v_{1}}^{\varepsilon_{1}} s_{0} a_{\mu_{1}}^{\varepsilon_{2}}$, где $v_{1}, \mu_{1} \notin\{1, \ldots, k\}, \varepsilon_{i}= \pm 1$, $i \in\{1,2\}$.

Доказательство очевидно.

Следствие 2. Пусть $F_{2}=\langle a, b\rangle-$ свободная группа ранга $2, H=\langle b, f\rangle-$ подгруппа группы $F_{2}, f \notin\langle b\rangle, f=a^{\varepsilon} f_{0} a^{r} b^{k}, \varepsilon, r \in\{ \pm 1\}$. Пусть для некоторого $z$, минимального в $H z H$ и удовлетворяющего условию $\mathrm{Hz} H \neq H \mathrm{H}$, и некоторого $b^{p}$ справедливо включение $z^{-1} b^{p} z \in H$, тогдa $z^{-1} b^{p} z=\left(a^{\varepsilon} f_{0} a^{r}\right)^{s}$, где $a^{\varepsilon} f_{0} a^{r} \equiv a^{\varepsilon} x b^{p} x^{-1} a^{-\varepsilon}, \varepsilon= \pm 1, s \in \mathbf{Z} \backslash\{0\}$.

Лемма 7. Пусть $F_{2}=\langle a, b\rangle-$ свободная группа ранга $2, H=\langle b, f\rangle$ - подгруппа группь $F_{2}, f=a^{\varepsilon_{1}} f_{0} a^{\varepsilon_{2}}, \varepsilon_{1} \neq 0, \varepsilon_{2} \neq 0, f \in\langle b\rangle$, u nycmb $w=\left(b^{k} a^{\mu}\right)^{r}, k \neq 0, \mu \neq 0, r \neq 0$. Пусть z минимален в $\mathrm{Hz} H, \mathrm{HzH} \neq \mathrm{HH}$.

Тогда 
- если $w^{*} \notin H$, но $z^{-1} w^{*} z \in H$, то $z^{-1} w^{*} z=f^{q}, q \in \mathbf{Z} \backslash\{0\}$;

- если существует циклическая перестановка $w^{*}$ слова $w$ такая, что $w^{*} \in H$, то $w^{*} \equiv f^{s}$, либо $w^{*}=\left(f b^{k}\right)^{s}$, либо $w^{*}=\left(b^{k} f\right)^{s}, s \in \mathbf{Z} \backslash\{0\}$.

Доказательство очевидно.

Следствие 3. Пусть $F_{2}$ и $H$ такие, как в лемме 7, $u w=a^{\mu}$. Тогда, если $a^{\mu} \in H$, то $a^{\mu}=f^{s}$, если $a^{\mu} \notin H u z$ минимально в $H z H, H z H \neq H H u z^{-1} a^{\mu} z \in H$, то $z^{-1} a^{\mu} z=f^{s}, s \in \mathbf{Z} \backslash\{0\}$.

Лемма 8. Пусть $H=\langle b, w\rangle$, где

$$
\begin{aligned}
& w \neq a x b^{k_{1}} x^{-1} a^{-1}, \\
& w \neq a x a^{k_{2}} x^{-1} a^{-1}, \\
& w \neq a^{\varepsilon} x\left(a^{\mu} b\right)^{ \pm p} x^{-1} a^{-\varepsilon} b^{-\mu}, \\
& w \neq a^{\varepsilon} x\left(a^{\mu} b^{-1}\right)^{ \pm p} x^{-1} a^{-\varepsilon} b^{\mu} .
\end{aligned}
$$

Тогда всякий аннулятор длинь, большей 3, однонаправлен.

Доказательство. Так как $\alpha_{-1}(C)=G$, то если неоднонаправленный аннулятор существует, то он имеет вид $\Sigma=(\bar{p}, \bar{c})$, где

$$
\bar{p}=\left(t, \ldots, h_{i-1}, t, h_{i}, t^{-1}, h_{i+1}, \ldots, t^{-1}\right) .
$$

Согласно леммам 2-5, группа $H$ не антинормальна, если $w=v^{p}$, либо $w b^{k}=v^{p},|p|>1$, поэтому рассмотрим следующие случаи.

1. Пусть $w=v^{p}$.

Предположим, что неоднонаправленный аннулятор существует, но тогда он обязан удовлетворять соотношению (3) и, следовательно, должно выполняться условие

$$
h_{i}^{-1} \alpha_{-1}\left(c_{i}\right) h_{i}=\alpha_{1}\left(c_{i+1}\right) \in H .
$$

По следствию 1 оно выполняется тогда и только тогда, когда

$$
\alpha_{-1}\left(c_{i}\right)=\left(v^{p}\right)^{s}, \quad \alpha_{1}\left(c_{i+1}\right)=\left(v^{p}\right)^{s} .
$$

Далее, чтобы аннулятор произвольной длины удовлетворял условию (3), должно выполняться соотношение

$$
h_{i+1}^{-1} t\left(v^{p}\right)^{s} t^{-1} h_{i+1}=h_{i+1}^{-1} b^{s} h_{i+1} \in H .
$$

Если $h_{i+1} \notin H$, то, выбирая $h_{i+1}$ минимальным в $H h_{i+1} H$, по следствию 2 получаем, что

$$
w=a^{\varepsilon} x b^{k_{1}} x^{-1} a^{-\varepsilon}
$$

и в этом случае существует неоднонаправленный аннулятор любой длины. Пусть

$$
w \neq a^{\varepsilon} x b^{k_{1}} x^{-1} a^{-\varepsilon}
$$

и $h_{i+1} \in H$. Для неоднонаправленного аннулятора произвольной длины должно выполняться условие

$$
h_{i+2}^{-1} t h_{i+1}^{-1} b^{s} h_{i+1} t^{-1} h_{i+2}=h_{i+2}^{-1}\left(h_{i+1}^{\prime}\right)^{-1} a^{s} h_{i+1}^{\prime} h_{i+2} \in H,
$$


где $h_{i+1}^{\prime}=t h_{i+1} t^{-1}$. По следствию 3 условие (4) справедливо тогда и только тогда, когда

$$
w=a^{\varepsilon} x a^{k_{2}} x^{-1} a^{-\varepsilon} .
$$

В этом случае может существовать неоднонаправленный аннулятор любой длины. Если

$$
w \neq a^{\varepsilon} x a^{k_{2}} x^{-1} a^{-\varepsilon}
$$

или

$$
w \neq a^{\varepsilon} x b^{k_{1}} x^{-1} a^{-\varepsilon},
$$

то предполагаемый аннулятор любой длины должен иметь вид

$$
\Sigma_{j}=\left(\bar{p}_{j}, \bar{c}_{j}\right), \quad j \in\{1,2\}
$$

где $p_{1}=\left(t, \ldots, t, h_{i}, t^{-1}, h_{i+1}, t^{-1}\right)$ и $p_{2}=\left(t, \ldots, t, h_{i}, t^{-1}\right)$. С другой стороны, если аннуляторы $\Sigma_{1}=\left(\bar{p}_{1}, \bar{c}_{1}\right)$ и $\Sigma_{2}=\left(\bar{p}_{2}, \bar{c}_{2}\right)$ существуют, то существуют и обратные к ним аннуляторы $\Sigma_{1}^{-1}=\left(\bar{p}_{1}^{-1}, \bar{c}_{1}^{-1}\right)$ и $\Sigma_{2}^{-1}=\left(\bar{p}_{2}^{-1}, \bar{c}_{2}^{-1}\right)$, где

$$
\bar{p}_{1}^{-1}=\left(t, h_{i+1}^{-1}, t, h_{i}^{-1}, t^{-1}, \ldots, t^{-1}\right), \quad \bar{p}_{2}^{-1}=\left(t, h_{i}^{-1}, t^{-1}, \ldots, t^{-1}\right) .
$$

Из вышеприведенных рассуждений следует, что число отрицательных $t$ в векторе $\bar{p}$ не может быть более двух, поэтому все неоднонаправленные аннуляторы исчерпываются множеством

$$
\left\{\Sigma_{1}=\left(\bar{p}_{1}, \bar{c}_{1}\right), \quad \Sigma_{2}=\left(\bar{p}_{2}, \bar{c}_{2}\right)\right\}
$$

где

$$
\bar{p}_{1}=\left(t, h_{-1}, t^{-1}, h_{0}, t^{-1}\right), \quad \bar{p}_{2}=\bar{p}_{1}^{-1} .
$$

Следовательно, кроме случаев, исключенных условием леммы, все аннуляторы длины более трех являются однонаправленными.

2. Пусть $w b^{ \pm k}=v^{p}, k \in \mathbf{N}$, где $w=a^{\varepsilon} w_{0} b^{\mu}$ и $\left(a^{\varepsilon} w_{0}\right)^{*} \notin\langle b\rangle$.

Рассмотрим подслучай $w b^{k}=v^{p}$.

Если предположить, что неоднонаправленный аннулятор существует, то, рассуждая аналогично вышеописанному, получаем, что должно выполняться равенство

$$
h_{i}^{-1}\left(w b^{k}\right)^{s} h_{i}=\left(w b^{k}\right)^{s}
$$

с точностью до сопряжения элементом $b^{k}$ (см. следствие 1).

Выясним теперь, в каких случаях

$$
h_{i+1}^{-1} t\left(w b^{k}\right)^{s} t^{-1} h_{i+1}=h_{i+1}^{-1}\left(b a^{k}\right)^{s} h_{i+1} \in H .
$$

Если любая циклическая перестановка $\left(\left(b a^{k}\right)^{s}\right)^{*} \notin H$, но вместе с тем

$$
h_{i+1}^{-1}\left(b a^{k}\right)^{s} h_{i+1} \in H,
$$

где $h_{i+1}$ минимально в $H h_{i+1} H$ и $H h_{i+1} H \neq H H$, то согласно первому утверждению леммы 7

$$
h_{i+1}^{-1}\left(\left(b a^{k}\right)^{s}\right)^{*} h_{i+1}=\left(w b^{k}\right)^{s},
$$

то есть, когда $w=a x\left(a^{k} b\right)^{s} x^{-1} a^{-1} b^{-k}$, либо $w=a x\left(a^{-k} b^{-1}\right)^{s} x^{-1} a^{-1} b^{-k}$, аннулятор произвольной длины может быть неоднонаправленным. Если существует циклическая перестановка $\left(\left(b a^{k}\right)^{s}\right)^{*} \in H$, то согласно второму утверждению леммы $7 w=\left(a^{k} b\right)^{s} b^{-k}$, либо 
$w=\left(a^{-k} b^{-1}\right)^{s} b^{-k}$. Во всех остальных случаях всякий аннулятор длины более трех может быть только однонаправленным.

В подслучае $w b^{-k}=v^{p}$ аналогично предыдущему подслучаю получаем, что неоднонаправленный аннулятор любой длины существует лишь тогда, когда либо $w=a x\left(a^{-k} b\right)^{s} x^{-1} a^{-1} b^{k}$, либо $w=a x\left(a^{k} b^{-1}\right)^{s} x^{-1} a^{-1} b^{k}$.

3. В тех случаях, когда $w=a w_{0} b^{k}$, группа $H$ порождается множеством $\left\langle b, a w_{0}\right\rangle$. Поэтому случаи, когда никакая циклическая перестановка $\left(a w_{0}\right)^{*}$ не принадлежит $\langle b\rangle$ относятся к вышеописанным. Рассмотрим случай, когда существует циклическая перестановка $\left(a w_{0}\right)^{*} \in\langle b\rangle$. Это возможно, если $w=a x b^{k_{1}} x^{-1} a^{-1}$, либо $w=a x b^{k^{\prime}} x^{-1} a^{-1} b^{k}$. Предположим, что неоднонаправленный аннулятор любой длины существует, тогда он обязан удовлетворять соотношению (3), то есть должно выполняться условие

$$
h_{i}^{-1} \alpha_{-1}\left(c_{i}\right) h_{i}=\alpha_{1}\left(c_{i+1}\right) \in H .
$$

В этом соотношении $h_{i}$ выбираем минимальным в $H h_{i} H$ и $H H \neq H h_{i} H$. В силу леммы 5 , если $h_{i} \neq(a x)^{ \pm 1}$ и $h_{i} \neq a x b^{l} x^{-1} a^{-1}$, где $l \in\left\{1, \ldots, k^{\prime}-1\right\}$, то

$$
h_{i}^{-1} H h_{i} \cap H=E
$$

и равенство (5) невозможно.

Пусть $h_{i}=a x$, тогда по лемме $3 \alpha_{1}\left(c_{i+1}\right) \in\left\langle b^{k^{\prime}}\right\rangle$, то есть $\alpha_{1}\left(c_{i+1}\right)=b^{k^{\prime}}$. Далее необходимо выполнение условия

$$
h_{i+1}^{-1} t b^{k^{\prime} s} t^{-1} h_{i+1} \in H,
$$

то есть $h_{i+1}^{-1} a^{k^{\prime}} h_{i+1} \in H$, что невозможно (см. леммы 3-5).

Пусть $h_{i}=a x b^{l} x^{-1} a^{-1}$, либо $h_{i}=(a x)^{-1}$. Тогда по лемме 4

$$
\alpha_{1}\left(c_{i+1}\right)=a x\left(b^{k^{\prime}}\right)^{s} x^{-1} a^{-1} .
$$

Далее, необходимо выполнение условия

$$
h_{i+1}^{-1} \operatorname{tax}\left(b^{k^{\prime}}\right)^{s} x^{-1} a^{-1} t h_{i+1}=h_{i+1}^{-1}\left(b a^{-k}\right)^{s} h_{i+1} .
$$

Так как любая циклическая перестановка $\left(\left(b a^{-k}\right)^{s}\right)^{*} \notin\left\langle b, a x b^{k^{\prime}} x^{-1} a^{-1}\right\rangle$, в силу первого утверждения леммы 7

$$
h_{i+1}^{-1}\left(b a^{-k}\right)^{s} h_{i+1}=\left(a x b^{k^{\prime}} x^{-1} a^{-1}\right)^{s_{1}},
$$

чего не может быть, так как число вхождений $a$ в левую часть равенства отлично от нуля, а в правую часть равенства равно нулю.

Таким образом, в этом случае всякий аннулятор длины более трех является однонаправленным.

Лемма доказана.

Продолжая доказательство теоремы 2, заметим, что группа $G=\langle a, b\rangle$ гиперболична как свободная группа ранга 2 , подгруппы $\alpha_{-1}(C), \alpha_{1}(C)$ квазивыпуклы в $G$ как подгруппы свободной группы. Теперь достаточно показать, что всякий существенный однонаправленный аннулятор длины $k, k \in \mathbf{N}$, с шириной, не большей $\rho$, и обхватом, не меньшим $H(\rho)$, является $\lambda$-гиперболическим. 
Рассмотрим следующие три возможности.

1. Пусть $G=\left\langle a, b, t ; t^{-1} a t=b, t^{-1} b t=a w_{0} a\right\rangle$.

Положим

$$
C=G=\langle a, b\rangle, \quad \alpha_{-1}(C)=C, \quad \alpha_{1}(C)=\langle b, w\rangle
$$

причем

$$
\alpha_{-1}(a)=a, \quad \alpha_{-1}(b)=b, \quad \alpha_{1}(a)=b, \quad \alpha_{1}(b)=w .
$$

Пусть $\varphi$ - эндоморфизм $\langle a, b\rangle \rightarrow\langle b, w\rangle$ такой, что $\varphi(a)=b, \varphi(b)=w$.

Пусть $f$ - свободноприведенное слово из $\langle a, b\rangle$. Так как $w^{ \pm 1}$ начинается и заканчивается соответственно символом $a^{ \pm 1}$, то $\varphi^{k}(f)$ - тоже свободноприведенное слово, кроме того, верны неравенства

$$
l\left(\varphi^{2}(f)\right) \geqslant l(w) l(f), \quad l(\varphi(f)) \leqslant l(w) l(f) .
$$

Заметим, что $l_{G}(f)=l_{C}(f)$, так как $G=C$. Положим $\lambda=3 / 2, H(\rho)=8 \rho l(w)$. Можем считать, что если $\Sigma=(\bar{p}, \bar{c})$, то

$$
\begin{array}{lll}
\bar{p}=\left(t, h_{-2}, t, h_{-1}, t, h_{0}, t, h_{1}, t\right), & h_{i} \in\langle a, b\rangle, & i \in\{-2, \ldots, 1\}, \\
\bar{c}=\left(c_{-2}, c_{-1}, c_{0}, c_{1}, c_{2}\right), & c_{i} \in C, & i \in\{-2, \ldots, 2\} .
\end{array}
$$

В силу выбора $\Sigma$

$$
l\left(c_{0}\right) \geqslant H(\rho)=8 \rho l(w), \quad l\left(h_{i}\right) \leqslant \rho, i \in\{-2, \ldots, 1\} .
$$

По определению аннулятора $h_{-1}^{-1} \varphi\left(c_{1}\right) h_{1}=c_{2}$. Следовательно,

$$
l\left(c_{2}\right) \geqslant l\left(\varphi\left(c_{1}\right)\right)-2 l\left(h_{1}\right) \geqslant l\left(\varphi\left(c_{1}\right)\right)-2 \rho .
$$

По определению аннулятора

$$
h_{0}^{-1} \varphi\left(c_{0}\right) h_{0}=c_{1} .
$$

Действуя на обе части равенства эндоморфизмом $\varphi$, получаем, что

$$
\varphi\left(h_{0}^{-1}\right) \varphi^{2}\left(c_{0}\right) \varphi\left(h_{0}\right)=\varphi\left(c_{1}\right) .
$$

Отсюда,

$$
l\left(\varphi\left(c_{1}\right)\right) \geqslant l\left(\varphi^{2}\left(c_{0}\right)\right)-2 l\left(\varphi\left(h_{0}\right)\right) .
$$

Из (6) следует, что

$$
2 l\left(\varphi\left(h_{0}\right)\right) \leqslant 2 l(w) l\left(h_{0}\right) \leqslant 2 l(w) \rho
$$

поэтому

$$
l\left(\varphi\left(c_{1}\right)\right) \geqslant l\left(\varphi^{2}\left(c_{0}\right)\right)-2 l(w) \rho .
$$

Используя еще раз соотношение (6), получаем, что

$$
l\left(\varphi^{2}\left(c_{0}\right)\right) \geqslant l(w) l\left(c_{0}\right)
$$

поэтому

$$
l\left(c_{2}\right) \geqslant l(w) l\left(c_{0}\right)-2(l(w)+1) \rho,
$$

и так как $l(w)>2$, то

$$
l\left(c_{2}\right) \geqslant(3 / 2) l\left(c_{0}\right)+(l(w)-3 / 2) l\left(c_{0}\right)-2(l(w)+1) \rho .
$$


Из соотношений $l(w)>2$ и $l\left(c_{0}\right) \geqslant H(\rho)=8 \rho l(w)$ получаем, что

$$
\begin{aligned}
l\left(c_{2}\right) & \geqslant \frac{3}{2} l\left(c_{0}\right)+\frac{1}{2} l\left(c_{0}\right)-2(l(w)+1) \rho \\
& \geqslant \frac{3}{2} l\left(c_{0}\right)+4 l(w) \rho-2 l(w) \rho-2 \rho>\frac{3}{2} l\left(c_{0}\right) .
\end{aligned}
$$

Таким образом, в данном случае всякий существенный аннулятор длины 5 , ширины, не большей $\rho$, и обхвата, не меньшего $H(\rho)$, является (3/2)-гиперболичным.

2. Пусть $G^{*}=\left\langle a, b, t ; t^{-1} a t=b, t^{-1} b t=a x w_{0} x^{-1} a^{-1}\right\rangle$, где $x=x(a, b),\left\|w_{0}\right\| \geqslant 2$.

Группа $C$ такая же, как и в предыдущем случае, а эндоморфизм $\varphi$ задается соотношениями $\varphi(a)=b, \varphi(b)=a \times w_{0} x^{-1} a^{-1}$.

Пусть $f$ - свободноприведенное слово от $a^{ \pm 1}$ и $b^{ \pm 1}$. Из того, что $w^{ \pm 1}$ начинается с $a$ и заканчивается на $a^{-1}$ следует, что в произведениях $\varphi(a) \varphi(b)$ или $\varphi(b) \varphi(a)$ нет сокращений, потому верны неравенства

$$
\begin{gathered}
l\left(\varphi^{2}(f)\right) \geqslant l\left(w_{0}\right) l(f), \\
l(\varphi(f)) \leqslant l(w) l(f) .
\end{gathered}
$$

Полагая $\lambda=3 / 2, H(\rho)=4 l(w) \rho+5 \rho$, аналогично предыдущему случаю, получаем, что

$$
l\left(c_{2}\right) \geqslant l\left(\varphi^{2}\left(c_{0}\right)\right)-2 l\left(\varphi\left(h_{0}\right)\right)-2 l\left(h_{1}\right) \geqslant l\left(\varphi^{2}\left(c_{0}\right)\right)-2 l\left(\varphi\left(h_{0}\right)\right)-2 \rho .
$$

Используя неравенство (8), получаем, что

$$
l\left(c_{2}\right) \geqslant l\left(\varphi^{2}\left(c_{0}\right)\right)-2 l(w) \rho-2 \rho .
$$

Используя неравенство (7), получаем оценку

$$
l\left(c_{2}\right) \geqslant l\left(w_{0}\right) l\left(c_{0}\right)-2 l(w) \rho-2 \rho .
$$

Так как $\left\|w_{0}\right\| \geqslant 2$, то $l\left(w_{0}\right) \geqslant 2$. Поэтому

$$
l\left(c_{2}\right) \geqslant 2 l\left(c_{0}\right)-2 l(w) \rho-2 \rho=\frac{3}{2} l\left(c_{0}\right)+\frac{1}{2} l\left(c_{0}\right)-2 l(w) \rho-2 \rho .
$$

Используя соотношение $l\left(c_{0}\right) \geqslant 4 l(w) \rho+5 \rho$, получаем, что

$$
l\left(c_{2}\right) \geqslant \frac{3}{2} l\left(c_{0}\right)+2 l(w) \rho+\frac{5}{2} \rho-2 l(w) \rho-2 \rho=\frac{3}{2} l\left(c_{0}\right)+\frac{1}{2} \rho,
$$

то есть $l\left(c_{2}\right)>(3 / 2) l\left(c_{0}\right)$.

Таким образом, всякий существенный аннулятор длины 5 , ширины, не большей $\rho$, и обхвата, не меньшего $H(\rho)$, является (3/2)-гиперболичным.

3. Пусть

$$
G^{*}=\left\langle a, b, t ; t^{-1} a t=b, t^{-1} b t=a^{m_{1}} w_{0} a^{m_{2}} b^{\mu}\right\rangle,
$$

где $w \neq a x\left(a^{\mu} b^{\delta}\right)^{p} x^{-1} a^{-1} b^{-\delta \mu}, \delta= \pm 1$ и $\left\|w_{0}\right\| \geqslant 2$, либо $w=a^{m} b^{n}$ и $|m| \geqslant|n|+2$.

В качестве группы $C$ возьмем группу, порожденную элементами $a$ и $\tilde{c}=b a^{-\mu}$. Пусть $\varphi$ - эндоморфизм вида $\varphi(a)=\tilde{c} a^{\mu}, \varphi(\tilde{c})=a^{m_{1}} w_{0} a^{m_{2}}$.

Пусть $f(a, b)$ - свободноприведенное слово в образующих $a$ и $b$ группы $G$. Обозначим $\tau(f(a, b))$ результат переписывания слова $f(a, b)$ в образующих группы $C$. Тогда $\tau\left(a^{m_{1}} w_{0} a^{m_{2}}\right)-$ слово $a^{m_{1}} w_{0} a^{m_{2}}$, записанное в образующих $a$ и $\tilde{c}$. 
Лемма 9. Пусть $G=\langle a, b\rangle-$ свободная группа ранга $2, f-$-свободноприведенное слово в алфавите $a^{ \pm 1}, b^{ \pm 1}$, и путь $v_{1} u v_{2}$ - элементы группы $G$ такие, что в произведениях $v_{1}^{\varepsilon} v_{2}^{\delta} v_{1}^{v} u v_{2}^{\varepsilon} v_{1}^{\delta} v_{2}^{v}$, где $\varepsilon, \delta, v= \pm 1$, слова $v_{2}^{\delta} u v_{1}^{\delta}$ в соответствующих произведениях не сокрапщаются полностью. Тогда слово $f^{\prime}$, полученное из $f$ подстановкой вместо каждого $a^{ \pm 1}$ слова $v_{1}^{ \pm 1}$, вместо каждого $b^{ \pm 1}$ слова $v_{2}^{ \pm 1}$, имеет длину, не меньшую длины слова $f$.

Доказательство леммы очевидно.

Продолжим рассмотрение случая 3. Нетрудно показать, что слова $\tau\left(a^{m_{1}} w_{0} a^{m_{2}}\right)$ и $\tilde{c} a^{\mu}$ удовлетворяют условиям леммы 9. В противном случае выполнялось бы либо равенство $a^{m_{1}} w_{0} a^{m_{2}} \equiv b^{-1} w_{0}$, либо $a^{m_{1}} w_{0} a^{m_{2}} \equiv w_{0} b$, чего не может быть.

Получаем, что

$$
l(\varphi(\tau(f(a, b)))) \geqslant l(\tau(f(a, b))) .
$$

Равенство в соотношении (9) достигается тогда и только тогда, когда

$$
\tau\left(a^{m_{1}} w_{0} a^{m_{2}}\right)=a^{-\mu} \tilde{c} .
$$

Рассмотрим слова $\varphi\left(a^{-\mu} \tilde{c}\right)=\left(\tilde{c} a^{\mu}\right)^{-\mu} a^{-\mu} \tilde{c}$ и $\varphi\left(\tilde{c} a^{\mu}\right)=a^{-\mu} \tilde{c}\left(\tilde{c} a^{\mu}\right)^{\mu}$. Видно, что при любых сокращениях от каждого из слов $\varphi\left(a^{-\mu} \tilde{c}\right)$ и $\varphi\left(\tilde{c} a^{\mu}\right)$ останутся по меньшей мере по две буквы, поэтому, так как $\varphi^{2}(a)=\varphi\left(\tilde{c} a^{\mu}\right)$ и $\varphi^{2}(\tilde{c})=\varphi\left(a^{-\mu} \tilde{c}\right)$, для этого случая верно неравенство

$$
l\left(\varphi^{2}(\tau(f))\right) \geqslant 2 l(\tau(f)) .
$$

Пусть теперь (9) есть строгое неравенство. Это значит, что от какого-то из слов $\tilde{c} a^{\mu}$ и $\tau\left(a^{m_{1}} w_{0} a^{m_{2}}\right)$ при всевозможных сокращениях остались минимум две буквы, но тогда справедливы неравенства

$$
\begin{gathered}
l(\varphi(\tau(f(a, b)))) \geqslant(3 / 2) l(\tau(f(a, b))), \\
l\left(\varphi^{2}(\tau(f(a, b)))\right) \geqslant(9 / 4) l(\tau(f(a, b))) .
\end{gathered}
$$

Объединяя неравенства (10) и (9), получим неравенство

$$
l\left(\varphi^{2}(\tau(f(a, b)))\right) \geqslant 2 l(\tau(f(a, b))) .
$$

Нетрудно также убедится в справедливости неравенства

$$
l(\varphi(\tau(f(a, b)))) \leqslant l(w) l(\tau(f(a, b))) .
$$

Выберем $\lambda=3 / 2, H(\rho)=4 l(w) \rho+5 \rho$. В силу выбора аннулятора $\Sigma=(\bar{p}, \bar{c})$

$$
\begin{array}{lll}
\bar{p}=\left(t, h_{-2}, t, h_{-1}, t, h_{0}, t, h_{1}, t\right), & h_{i} \in\langle a, b\rangle, & i \in\{-2, \ldots, 1\}, \\
\bar{c}=\left(c_{-2}, c_{-1}, c_{0}, c_{1}, c_{2}\right), & c_{i} \in C, & i \in\{-2, \ldots, 2\},
\end{array}
$$

причем $l\left(c_{0}\right) \geqslant H(\rho)$ и $l\left(h_{i}\right) \leqslant \rho$ для $i \in\{-2, \ldots, 1\}$. По определению аннулятора $h_{-1}^{-1} \varphi\left(c_{1}\right) h_{1}=c_{2}$. Следовательно,

$$
l\left(c_{2}\right) \geqslant l\left(\varphi\left(c_{1}\right)\right)-2 l\left(h_{1}\right) \geqslant l\left(\varphi\left(c_{1}\right)\right)-2 \rho .
$$

Аналогично, из определения аннулятора следует, что $h_{0}^{-1} \varphi\left(c_{0}\right) h_{0}=c_{1}$. Действуя на обе части равенства эндоморфизмом $\varphi$, находим, что

$$
\varphi\left(h_{0}^{-1}\right) \varphi^{2}\left(c_{0}\right) \varphi\left(h_{0}\right)=\varphi\left(c_{1}\right) .
$$


Отсюда получаем неравенство

$$
l\left(\varphi\left(c_{1}\right)\right) \geqslant l\left(\varphi^{2}\left(c_{0}\right)\right)-2 l\left(\varphi\left(h_{0}\right)\right) .
$$

Из соотношения (13) следует, что

$$
2 l\left(\varphi\left(h_{0}\right)\right) \leqslant 2 l(w) l\left(h_{0}\right) \leqslant 2 l(w) \rho,
$$

откуда

$$
l\left(\varphi\left(c_{1}\right)\right) \geqslant l\left(\varphi^{2}\left(c_{0}\right)\right)-2 l(w) \rho .
$$

Из неравенства (12) следует, что $l\left(\varphi^{2}\left(c_{0}\right)\right) \geqslant 2 l\left(c_{0}\right)$, поэтому

$$
l\left(c_{2}\right) \geqslant 2 l\left(c_{0}\right)-2(l(w)+1) \rho,
$$

откуда

$$
l\left(c_{2}\right) \geqslant(3 / 2) l\left(c_{0}\right)+\left((1 / 2) l\left(c_{0}\right)-2(l(w)+1) \rho\right),
$$

то есть

$$
\begin{aligned}
& l\left(c_{2}\right) \geqslant(3 / 2) l\left(c_{0}\right)+2 l(w) \rho+(5 / 2) \rho-2 l(w) \rho-2 \rho=(3 / 2) l\left(c_{0}\right)+(1 / 2) \rho, \\
& l\left(c_{2}\right)>(3 / 2) l\left(c_{0}\right) .
\end{aligned}
$$

Таким образом, всякий существенный аннулятор длины 5 , ширины, не большей $\rho$ и обхвата, не меньшего $H(\rho)$, является (3/2)-гиперболичным, следовательно, по теореме Бествины рассматриваемые группы являются гиперболическими.

4. Пусть теперь

$$
G^{*}=\left\langle a, b, t ; t^{-1} a t=b, t^{-1} b t=a^{m} b^{n}\right\rangle,
$$

где $|m|<|n|+1$ и $|m| \neq|n|-1$ и $|m| \neq 1$.

Введем обозначение $H=\langle b, w\rangle$, где $w=a^{m} b^{n}, H_{i}=t^{-i} H t^{i}$. Пусть $f-$ свободноприведенное слово в группе $G=\langle a, b\rangle$ и $\varphi: G \rightarrow G-$ эндоморфизм такой, что $\varphi(a)=b$, $\varphi(b)=w$. Оценим длину слова

$$
\varphi(f)=\varphi\left(a^{x_{1}} b^{y_{1}} a^{x_{1}} b^{y_{2}} \ldots a^{x_{j}} b^{y_{j}}\right) .
$$

Введем обозначения

$$
\begin{aligned}
& x=\left|x_{1}\right|+\left|x_{2}\right|+\ldots+\left|x_{j}\right|, \\
& y=\left|y_{1}\right|+\left|y_{2}\right|+\ldots+\mid y_{j} .
\end{aligned}
$$

Тогда $l(f)=x+y$. Очевидно, что в слове $\varphi(f)$ сократиться может только некоторая степень $b$. Поэтому, если $x \geqslant|n| y$, то

$$
\begin{aligned}
l(\varphi(f)) & \geqslant x+y(|m|+|n|)-2|n| j \\
& \geqslant x+y(|m|+|n|)-2|n| y=x_{1}+y|m|,
\end{aligned}
$$

где $x=x_{1}+|n| y$, и если $x<|n| y$, то

$$
l(\varphi(f)) \geqslant x+y(|m|+|n|)-2 x>y|m| .
$$

Поэтому

$$
\frac{l(\varphi(f))}{l(f)}=\frac{x_{1}+y|m|}{x+y}=\frac{x_{1}+y|m|}{x_{1}+y(|n|+1)} .
$$


Так как $|m|<|n|+1$, то

$$
\frac{x_{1}+y|m|}{x_{1}+y(|n|+1)}>\frac{y|m|}{y(|n|+1)}
$$

Тогда

$$
\frac{l(\varphi(f))}{l(f)}>\frac{y|m|}{y(|n|+1)}=\frac{|m|}{|n|+1} .
$$

Таким образом,

$$
l(\varphi(f))>\frac{|m|}{|n|+1} l(f) .
$$

Из тех же соображений получаем, что

$$
l\left(\varphi^{2}(f)\right) \geqslant\left(\frac{|m|}{|n|+1}\right)^{2} l(f) .
$$

Рассмотрим группу $H_{1}=\left\langle w, w_{1}\right\rangle$, где $w_{i}=t^{-i} w t^{i}$. Нетрудно показать, что всякое свободноприведенное слово $f_{1} \in H_{1}$ есть слово от элементов $a^{ \pm m}, b^{\alpha}$, где $\alpha \in\{ \pm m, \pm n, \pm(n \pm m)\}$, причем либо $n \pm m=0$, либо $n \pm m \geqslant 2$.

Пусть $l\left(f_{1}\right)=x+y$, где $x$ - длина по $a, y$ - длина по $b$. Тогда если

$$
f_{1}=\left(a^{m}\right)^{x_{1}} \ldots\left(b^{\alpha}\right)^{y_{j}}
$$

то $x$ кратно $m$, а $y \geqslant 2 j$.

Оценим длину слова $\varphi^{2}\left(f_{1}\right)$. Поскольку $\varphi^{2}\left(f_{1}\right)$ есть слово из элементов $\left(a^{m} b^{n}\right)^{m}$ и $\left(b^{m}\left(a^{m} b^{n}\right)^{n}\right)^{\alpha}$, в слове $\varphi^{2}\left(f_{1}\right)$ максимальное сократимое подслово имеет вид $\left(b^{m}\left(a^{m} b^{n}\right)^{n}\right)$. Тогда

$$
l\left(\varphi^{2}\left(f_{1}\right)\right) \geqslant x(|m|+|n|)+y(|m|+|n|)|n|+y|m|-2 j((|m|+|n|)|n|+|m|) .
$$

Рассмотрим следующие два случая.

Пусть $j=y / 2$. Справедливо неравенство

$$
2 x \geqslant y \text {. }
$$

Действительно, максимальное значение для $y$ есть $y=2 j$, а минимальное значение для $x$ есть $x=2 j$, если $f_{1}=\left(a^{m}\right)^{x_{1}} \ldots\left(b^{\alpha}\right)^{y_{j}}$, либо $x=2(j-1)$, если $f=\left(b^{\alpha}\right)^{y_{1}}\left(a^{m}\right)^{x_{1}} \ldots\left(b^{\alpha}\right)^{y_{j}}$, и так как при $j \geqslant 2$ неравенство $4(j-1) \geqslant 2 j$ справедливо, справедливо и неравенство (16). Тогда

$$
\begin{aligned}
l\left(\varphi^{2}\left(f_{1}\right)\right) & >x(|m|+|n|)+y(|m|+|n|)|n|+y|m|-y((|m|+|n|)|n|+|m|) \\
& =x(|m|+|n|)
\end{aligned}
$$

и

$$
\frac{l\left(\varphi^{2}\left(f_{1}\right)\right)}{l\left(f_{1}\right)}>\frac{x(|m|+|n|)}{x+y}>\frac{x(|m|+|n|)}{2 x+x}=\frac{|m|+|n|}{3} \geqslant 4 / 3 .
$$

Пусть теперь $j<y / 2$. Представим $y$ как $y=y_{1}+2 j$, где $2 x \geqslant 2 j$, тогда

$$
l\left(\varphi^{2}\left(f_{1}\right)\right)>x(|m|+|n|)+y_{1}((|m|+|n|)|n|+|m|)
$$

3 Дискретная математика, т.14 №3 
и

$$
\begin{aligned}
\frac{l\left(\varphi^{2}\left(f_{1}\right)\right)}{l\left(f_{1}\right)} & >\frac{x(|m|+|n|)+y_{1}((|m|+|n|)|n|+|m|)}{x+y_{1}+2 j} \\
& >\frac{x(|m|+|n|)}{x+2 j} \geqslant 4 / 3 .
\end{aligned}
$$

Таким образом, $l\left(\varphi^{2}\left(f_{1}\right)\right)>(4 / 3) l\left(f_{1}\right)$. Из тех же соображений следует, что

$$
l\left(\varphi^{2 k}\left(f_{1}\right)\right)>(4 / 3)^{k} l\left(f_{1}\right) .
$$

Объединяя неравенства (17) и (18), получим, что

$$
l\left(\varphi^{2 k}(f)\right)>\left(\frac{4}{3}\right)^{k}\left(\frac{|m|}{|n|+1}\right)^{2} l(f)
$$

где $f_{1}=\varphi^{2}(f)$. Поэтому при

$$
k \geqslant \log _{4 / 3}\left(2\left(\frac{|n|+1}{m}\right)^{2}\right)+1
$$

справедлива оценка

$$
l\left(\varphi^{2 k}(f)\right)>2 l(f)
$$

Положим теперь

$$
C=G=\langle a, b\rangle, \quad \alpha_{-1}(C)=C, \quad \alpha_{1}(C)=H,
$$

причем $\alpha_{-1}(a)=a, \alpha_{-1}(b)=b, \alpha_{1}(a)=b, \alpha_{1}(b)=w$. Кроме того считаем, что

$$
l(\varphi(f)) \leqslant l(w) l(f) .
$$

Заметим, что $l_{G}(f)=l_{C}(f)$, так как $G=C$. ПІоложим

$$
\lambda=\frac{3}{2}, \quad H(\rho)=16 \rho \frac{l^{2 k}(w)-1}{l(w)-1},
$$

и так как любой аннулятор длины, большей трех, однонаправлен, считаем, что $\Sigma=(\bar{p}, \bar{c})$, где

$$
\begin{array}{ll}
\bar{p}=\left(t, h_{-2 k}, t, h_{-2 k+1}, t, \ldots, t, h_{0}, \ldots, t, h_{2 k-1}, t\right), & h_{i} \in G, \quad i \in\{-2 k, \ldots, 2 k-1\}, \\
\bar{c}=\left(c_{-2 k}, c_{-2 k+1}, \ldots, c_{0}, \ldots, c_{2 k}\right), & c_{i} \in C, \quad i \in\{-2 k, \ldots, 2 k\} .
\end{array}
$$

В силу выбора $\Sigma$

$$
l\left(c_{0}\right) \geqslant H(\rho), \quad l\left(h_{i}\right) \leqslant \rho, \quad i \in\{-2 k, \ldots, 2 k-1\} .
$$

Из определения аннулятора следует, что

$$
h_{2 k-1}^{-1} \varphi\left(c_{2 k-1}\right) h_{2 k-1}=c_{2 k}
$$


Следовательно,

$$
l\left(c_{2 k}\right) \geqslant l\left(\varphi\left(c_{2 k-1}\right)\right)-2 l\left(h_{2 k-1}\right) \geqslant l\left(\varphi\left(c_{2 k-1}\right)\right)-2 \rho .
$$

Аналогично, из определения аннулятора следует, что

$$
h_{2 k-2}^{-1} \varphi\left(c_{2 k-2}\right) h_{2 k-2}=c_{2 k-1} \text {. }
$$

Действуя на обе части равенства эндоморфизмом $\varphi$, получаем, что

$$
\varphi\left(h_{2 k-2}^{-1}\right) \varphi^{2}\left(c_{2 k-2}\right) \varphi\left(h_{2 k-2}\right)=\varphi\left(c_{2 k-1}\right) .
$$

Поэтому верно неравенство

$$
l\left(\varphi\left(c_{2 k-1}\right)\right) \geqslant l\left(\varphi^{2}\left(c_{2 k-2}\right)\right)-2 l\left(\varphi\left(h_{2 k-2}\right)\right) .
$$

Из определения аннулятора следует, что

$$
h_{2 k-3}^{-1} \varphi\left(c_{2 k-3}\right) h_{2 k-3}=c_{2 k-2} \text {. }
$$

Действуя на обе части равенства эндоморфизмом $\varphi^{2}$, получим равенство

$$
\varphi^{2}\left(h_{2 k-3}^{-1}\right) \varphi^{3}\left(c_{2 k-3}\right) \varphi^{2}\left(h_{2 k-3}\right)=\varphi^{2}\left(c_{2 k-2}\right) .
$$

Следовательно,

$$
l\left(\varphi^{2}\left(c_{2 k-2}\right)\right) \geqslant l\left(\varphi^{3}\left(c_{2 k-3}\right)\right)-2 l\left(\varphi^{2}\left(h_{2 k-3}\right)\right) .
$$

Рассуждая аналогичным образом, получаем неравенства

$$
\begin{aligned}
l\left(\varphi^{3}\left(c_{2 k-3}\right)\right) & \left.\geqslant l\left(\varphi^{4}\left(c_{2 k-4}\right)\right)-2 l\left(\varphi^{3} h_{2 k-4}\right)\right), \\
& \ldots \\
l\left(\varphi^{2 k-1}\left(c_{1}\right)\right) & \geqslant l\left(\varphi^{2 k}\left(c_{0}\right)\right)-2 l\left(\varphi^{2 k-1}\left(h_{0}\right)\right) .
\end{aligned}
$$

Подставляя теперь в неравенство (21) неравенство (22), получим, что

$$
\left.l\left(c_{2 k}\right) \geqslant l\left(\varphi^{2}\left(c_{2 k-2}\right)\right)-2 l\left(\varphi\left(h_{2 k-2}\right)\right)\right)-2 \rho .
$$

Затем, подставляя в неравенство (25) последовательно неравенство (23) и неравенства (24), получим неравенство

$$
\begin{aligned}
l\left(c_{2 k}\right) \geqslant l\left(\varphi^{2 k}\left(c_{0}\right)\right)-2 l\left(\varphi^{2 k-1}\left(h_{0}\right)\right) & \\
& -2 l\left(\varphi^{2 k-2}\left(h_{1}\right)\right)-\ldots-2 l\left(\varphi^{2}\left(h_{2 k-3}\right)\right)-2 l\left(\varphi\left(h_{2 k-2}\right)\right)-2 \rho .
\end{aligned}
$$

Используя неравенства (19) и (20), получим неравенство

$$
\begin{aligned}
\left.\left.l\left(c_{2 k}\right)>2 l\left(c_{0}\right)\right)-2 l^{2 k-1}(w) l\left(h_{0}\right)\right) & \\
& \left.\left.\left.-2 l^{2 k-2}(w) l\left(h_{1}\right)\right)-\ldots-2 l^{2}(w) l\left(h_{2 k-3}\right)\right)-2 l(w) l\left(h_{2 k-2}\right)\right)-2 \rho .
\end{aligned}
$$

Учитывая неравенства $l\left(h_{i}\right) \leqslant \rho$, получим, что

$$
l\left(c_{2 k}\right) \geqslant 2 l\left(c_{0}\right)-2 \rho \frac{l^{2 k}(w)-1}{l(w)-1} .
$$


Используя неравенство

$$
l\left(c_{0}\right) \geqslant H(\rho)=16 \rho \frac{l^{2 k}(w)-1}{l(w)-1},
$$

получим, что

$$
\begin{aligned}
l\left(c_{2 k}\right) & \geqslant \frac{3}{2} l\left(c_{0}\right)+8 \rho \frac{l^{2 k}(w)-1}{l(w)-1}-2 \rho \frac{l^{2 k}(w)-1}{l(w)-1} \\
& =\frac{3}{2} l\left(c_{0}\right)+6 \rho \frac{l^{2 k}(w)-1}{l(w)-1}>\frac{3}{2} l\left(c_{0}\right) .
\end{aligned}
$$

Таким образом, всякий аннулятор длины $4 k+1$ является (3/2)-гиперболическим.

Теорема 2 доказана.

Доказательство теоремы 3. Используя тот факт, что гиперболическая группа не содержит подгруппу, изоморфную группе Баумслага

$$
G(m, n)=\left\langle a, t ; t^{-1} a^{m} t=a^{n}\right\rangle,
$$

где $m \neq 0, n \neq 0$ (см. [11]), либо 2-порожденную абелеву подгруппу (см. [12]), нетрудно показать, что если

$$
w \in\left\{a x b^{k_{1}} x^{-1} a^{-1}, a x a^{k_{2}} x^{-1} a^{-1}, a x\left(a^{\mu} b^{\delta}\right)^{p} x^{-1} a^{-1} b^{-\delta \mu}\right\},
$$

где $\delta= \pm 1$, то группа $G^{*}$ содержит подгруппу, изоморфную группе Баумслага. Пусть, к примеру,

$$
w=a x\left(a^{\mu} b\right)^{p} x^{-1} a^{-1} b^{-\mu} .
$$

Тогда, так как $t^{-1} b a^{\mu} t=a x\left(a^{\mu} b\right)^{p} x^{-1} a^{-1}$, положив $b a^{\mu}=b^{\prime}, d=t a x b^{-1}$, получим, что $G^{*}$ содержит подгруппу

$$
\left\langle b^{\prime}, d ; d^{-1} b^{\prime} d=\left(b^{\prime}\right)^{p}\right\rangle
$$

Пусть теперь

$$
w=a x\left(a^{\mu} b\right)^{ \pm p} x^{-1} a^{-1} b^{-\mu},
$$

тогда при $p=1$ и $a x=1$ получаем, что $w=a^{\mu} b^{1 \pm \mu}$. Если

$$
w=a x\left(a^{\mu} b^{-1}\right)^{ \pm p} x^{-1} a^{-1} b^{\mu},
$$

то при $p=1$ и $a x=1$ получаем, что $w=a^{\mu} b^{ \pm \mu-1}$.

Таким образом, когда $w=a^{m} b^{n}$, где $|m|=|n| \pm 1$, рассматриваемые группы не гиперболические.

Рассмотрим группу

$$
G^{*}=\left\langle a, b, t ; t^{-1} a t=b, t^{-1} b t=a^{ \pm 1} b^{n}\right\rangle
$$

В случае $w=a b^{n}$ группа $G^{*}$ содержит абелеву подгруппу, а именно,

$$
\left\langle b a b^{-1} a^{-1}, t^{2} ; t^{-2} b a b^{-1} a^{-1} t^{2}=b a b^{-1} a^{-1}\right\rangle .
$$

Действительно,

$$
\begin{aligned}
t^{-1} b a b^{-1} t & =a b^{n} b b^{-n} a^{-1}=a b a^{-1} \\
t^{-1} b a^{-1} b^{-1} t & =a b^{n} b^{-1} b^{-n} a^{-1}=a b^{-1} a^{-1} .
\end{aligned}
$$


Поэтому

$$
t^{-2} b a b a^{-1} t^{2}=t^{-1} a b a^{-1} b^{-1} t=t^{-1} a t t^{-1} b a^{-1} b^{-1} t=b a b^{-1} a^{-1} .
$$

В случае $w=a^{-1} b^{n}$ группа $G^{*}$ содержит абелеву подгруппу, а именно,

$$
\left\langle b a b^{-1} a^{-1}, t^{2} b^{-1} a^{-1} ;\left(t^{2} b^{-1} a^{-1}\right)^{-1} b a b^{-1} a^{-1}\left(t^{2} b^{-1} a^{-1}\right)=b a b^{-1} a^{-1}\right\rangle .
$$

Действительно,

$$
t^{-1} b a b^{-1} t=a^{-1} b^{n} b b^{-n} a=a^{-1} b a .
$$

Поэтому

$$
\begin{aligned}
a b t^{-2} b a b^{-1} a^{-1} t^{2} b^{-1} a^{-1} & =a b t^{-1} a^{-1} b a b^{-1} t b^{-1} a^{-1} \\
& =a b b^{-1} a^{-1} b a b^{-1} a^{-1}=b a b^{-1} a^{-1}
\end{aligned}
$$

Теорема доказана.

\section{Список литературы}

1. Лысенок И. Г., О некоторых алгоритмических свойствах гиперболических групп. Изв. $А Н C С C P$, Сер. мат. (1989) 53, №4, 814-832.

2. Григорчук Р. И., Курчанов П. Ф., Некоторые вопросы теории групп, связанные с геометрией. Итоги науки и техн. Совр. пробл. матем. Фунд. напр. (1990) 58, 191-256.

3. Ольшанский А. Ю., $S Q$-универсальность гиперболических групп. Матем. сб. (1995) 186, №8, 119-132.

4. Ivanov S. V., Schupp P. E., On the hyperbolicity of small concellation groups and one-relator groups. Trans. Amer. Math. Soc. (1999) 350, №5, 1851-1894.

5. Kapovich I., Howson property and one-relator groups. Commun. Algebra (1999) 27, №3, 1057-1072.

6. Bestvina M., Feighn M., The combination theorem for negatively curved groups. J. Diff. Geometry (1992) 35, №1, 85-101.

7. Bestvina M., Feighn M., Addendum and correction to: "A combination theorem for negatively curved groups.” J. Diff. Geom. (1996) 43, №4, 783-788.

8. Щепетьева Н. Б., Гиперболичность некоторых двупорожденных групп с одним определяющим соотношением. В сб.: Всероссийская науч. конф. “Современные проблемы математики, механики и информатики”, посв. 70-летию ТулГу. Тезисы докладов. Тульский госунив., Тула, 2000, c. 64-65.

9. Линдон Р., Шупп П., Комбинаторная теория групп. Мир, Москва, 1980.

10. Безверхний В. Н., Решение проблем сопряженности слов в одном классе групп. В сб.: Алгоритмич. проблемы теории групп и подгрупп. Межвуз. сб. Тула, 1997, с. 4-38.

11. Gersten S. M., Short H., Rational subgroups of biautomatic groups. Ann. Math. (1991) 134, 125-158.

12. Gromov M., Hyperbolic groups. In: Essays in group theory. Springer, Berlin, 1987, 75-263.

13. Alonso I., Brady T., Cooper D., Ferlini V. , Lustig M., Mihalik M., Shapiro M., Short H., Notes on hyperbolic groups. Group theory from a geometric view point. In: Proc. ICTP Trieste. World Scientific, Singapore, 1991, pp. 3-63. 